\title{
Philosophiques
}

\section{Lire Bergson (Réponse à J. Goulet)}

\section{Guy Lafrance}

Volume 3, numéro 2, octobre 1976

URI : https://id.erudit.org/iderudit/203057ar

DOI : https://doi.org/10.7202/203057ar

Aller au sommaire du numéro

Éditeur(s)

Société de philosophie du Québec

ISSN

0316-2923 (imprimé)

1492-1391 (numérique)

Découvrir la revue

Citer ce document

Lafrance, G. (1976). Lire Bergson (Réponse à J. Goulet). Philosophiques, 3(2),

279-284. https://doi.org/10.7202/203057ar

Ce document est protégé par la loi sur le droit d'auteur. L'utilisation des services d'Érudit (y compris la reproduction) est assujettie à sa politique d'utilisation que vous pouvez consulter en ligne.

https://apropos.erudit.org/fr/usagers/politique-dutilisation/ 


\title{
LIRE BERGSON
}

\author{
(Réponse à J. Goulet) \\ par Guy Lafrance
}

La lecture de l'étude critique faite par $M$. Jean Goulet de mon ouvrage sur La philosophie sociale de Bergson a suscité chez moi et chez de nombreux lecteurs de la Revue, qui ont tenu à me faire part de leur réaction, un vif sentiment d'indignation. Indignation devant le ton cavalier et même grossier de l'article et son style de mauvais goût. Indignation aussi devant cette façon superficielle de regarder " la forme " d'un ouvrage à partir de sa table de matière et d'en rester là, pour en déduire à partir du nombre de pages consacrées à chacun des thèmes traités, l'importance que l'auteur accorde à ceux-ci! Indignation enfin devant l'impertinence générale de cette critique qui se permet des ratés considérables dans la pensée même de Bergson qu'elle extrapole plus qu'elle ne comprend, des ratés du même genre dans la lecture de notre texte que $M$. Goulet, volontairement ou involontairement je ne saurais dire, déforme du tout au tout par des inversions évidentes du sens des phrases tirées hors de leur contexte; le tout assaisonné d'un prêchi-prêcha alimenté d'une certaine métaphysique, et de l'assurance pour ne pas dire de l'outrecuidance trop évidente du défenseur offensé de la vérité.

Une telle critique ne mérite pas de réponse pour elle-même car elle ne donne prise à aucun élément de discussion sensée sur la pensée de Bergson. Comme, en outre, cette critique soulève un doute évident sur la lecture même de notre ouvrage faite par $M$. Goulet, qui semble s'en être tenu à glaner ici et là quelques phrases pour servir de point de départ à ses extrapolations, plutôt qu'à une lecture motivée par une recherche interne du sens, nous nous sentions tout à fait légitimé de ne pas donner suite à ce genre d'étude.

Pourtant, toute chose a ses limites. On ne peut pas et on ne doit pas, surtout à l'intérieur d'une nouvelle Revue philosophique 
qui entend servir d'outil à une jeunesse intellectuelle encore fragile à l'intérieur d'une société non moins fragile, laisser librement et tacitement cours à l'arbitraire et au dogmatisme étroit sans qu'on en subisse la conséquence. C'est cette unique raison, soucieuse me dira-t-on de la qualité de notre production philosophique collective, qui m'a incité à prendre la plume.

Je veux d'abord, par cette intervention, dénoncer ce style dé. plorable de la critique philosophique qui vise de façon évidente la destruction systématique, arbitraire et inconditionelle, qui ne débouche sur rien de constructif et qui est peut-être la conséquence de l'aigreur et des frustrations ressenties par ces personnes (et elles sont légion chez nous) qui prennent subitement et tardivement conscience de la pauvreté ou de l'absence pure et simple de leur production philosophique, qu'elles cherchent désespérément à justifier ou à valoriser en utilisant avec une totale irresponsabilité l'outil de la destruction.

Je veux surtout, puisqu'il s'agit ici d'une lecture de Bergson et des études bergsoniennes, dénoncer ce genre d'interprétation d'allure poétique et extrapolante qui passe par toutes les transformations du prisme et qui préfère lire à travers les phrases de Bergson les idées qu'ils y mettent eux-mêmes plutôt que d'en lire le message authentique de Bergson par crainte d'y voir déranger leurs propres convictions. Une lecture tant soit peu bergsonienne de Bergson, c'est-à-dire d'un Bergson dont le fil de la pensée commande d'être suivi de l'intérieur et qui dévoile par là même sa perméabilité aux influences extérieures par son souci d'ajustement continuel à un réel qui bouge sans cesse, - et cela s'entend à plus forte raison de l'ensemble du climat intellectuel à l'intérieur duquel Bergson a développé et véhiculé sa pensée, - devrait suffire à décourager au point de départ toute interprétation dogmatique, qui vise à isoler Bergson sur lui-même comme s'il avait besoin d'être protégé de la sorte pour être mieux défendu, et qui crie au scandale en brandissant l'épithète infamante de "positivisme ", dès qu'on lui montre un Bergson en son temps, curieux de toucher du doigt la réalité sous ses multiples facettes et qui n'a pas craint par souci de positivité philosophique de se mettre aux écoutes des sciences positives.

M. Goulet, à travers sa critique, offre un exemple patent d'une lecture fermée de Bergson quand il écrit: «il y a lieu 
d'espérer qu'on va encore relire Bergson sérieusement, comme un " philosophe digne de ce nom » qu'il faut préserver, non seulement par rapport aux sciences de la nature, mais aussi pour sa contribution aux sciences humaines" (p. 86). Outre son incohérence notoire, cette phrase est une véritable injure à la pensée de Bergson, car jamais Bergson n'aurait accepté qu'on cherchât à "préserver " sa pensée par rapport aux sciences, que ce soit les sciences de la nature ou les sciences sociales. Toute sa démarche philosophique en a été une essentiellement de confrontation avec les sciences dont il voulait découvrir à la fois la richesse et les limites pour mieux camper sa réflexion philosophique. Bergson n'a pas eu peur du caractère positif des sciences; il a eu le courage de se mettre à leurs écoutes et de les étudier pour en découvrir leur richesse et leur valeur de vérité.

M. Goulet a manifestement peur de lire Bergson à travers ses exigences de positivité et de confrontations qu'il jugeait nécessaires avec la science de son temps. M. Goulet passe même aux aveux d'incompétence en ce domaine quand il dit : « je laisse à des sociologues de métier le soin de ...... ou encore "il y aurait lieu de réexaminer point par point la comparaison instructive que l'auteur conduit, avec beaucoup de succès, et pour cause, entre les divers aspects de la société close, selon Bergson, et ce que la sociologie d'alors en dit " (p. 87). Tout ceci témoigne de façon évidente d'un double échec.

Échec dans la saisie de la pensée de Bergson d'abord et à plus forte raison de sa pensée sociale, qui contient cette référence indispensable aux sciences et en particulier aux sciences sociales de son époque. Échec aussi dans la saisie de notre interprétation de la pensée sécoale de Bergson puisqu'un des buts premiers et explicites de notre ouvrage est de mettre en lumière cette pensée sociale de Bergson qui se forme dans un contexte intellectuel vivant et qui s'est mesurée nécessairement avec les idées sociologiques et anthropologiques qui avaient cours à l'époque. $M$. Goulet n'a rien compris de tout cela et ne semble même pas s'en être rendu compte.

Serait-ce cette même peur du positivisme qui amène $M$. Goulet à ne pas lire correctement le sens de nos phrases ? Quand il dit en nous citant de travers " pour celui qui dénie à son entreprise philosophique de vouloir aller plus loin que les (...) sciences" (p. 
92) ; il aurait dû d'abord nous citer correctement afin de mieux nous comprendre. Nous disions : "Elle (l'entreprise philosophique pour Bergson) ne devait pas se présenter comme une sythèse des autres sciences, encore moins comme une démarche pour aller plus loin que les diverses sciences en empruntant la même direction qu'elles " (p. 136). En ne retenant qu'un membre de la phrase, $M$. Goulet effectue une grossière inversion du sens.

Les lecteurs avertis de Bergson savent d'ailleurs que celui-ci n'a jamais prétendu dépasser la science en s'engageant sur le même terrain qu'elle. Ce genre de prétention constituait aux yeux de Bergson une injure à la science. Il estimait, bien sûr, que la philosophie avait un domaine propre de recherche et qu'elle pouvait sur son propre terrain dépasser les conclusions de la science, mais dans une autre direction. Il n'y a pas d'opposition ou de conflit, pour Bergson, entre la science et la philosophie, mais complémentarité. Comment une lecture sensée de Bergson peut-elle conduire à ces dichotomies irréductibles?

C'est encore une lecture faussée de Bergson qui amène $M$. Goulet à faire des sommations du genre " il faut choisir "entre l'émotion qui est le ressort de la morale bergsonienne et "la pure positivité qui, elle, pour être comprise, requiert qu'on ne s'émeuve jamais " (p. 88), sic. Quel mépris à l'égard de la positivité scientifique, dans laquelle Bergson n'hésitait pas à reconnaître qu'elle était chargée d'émotion créatrice ! Et quelle attitude des plus contraire à la pensée même de Bergson qui jusque dans ses oppositions en apparence les plus radicales a voulu montrer la conciliation possible à travers la continuité !

Puisque $M$. Goulet a cru devoir choisir un élément de sa fausse dichotomie, celui qui répond sans doute à ses convictions personnelles, il se laisse prendre à son propre jeu en tentant de faire voir, en termes à peine voilés à la fin de son texte, un Bergson apologète de la foi chrétienne ! Il y a de quoi désespérer à lire pareil discours quand on sait avec quelle précaution Bergson s'est aventuré dans les questions religieuses, en les abordant par leurs manifestations les plus tangibles à travers ces grands hommes d'action que sont les mystiques. Mais ce sont ces actions créatrices des héros et des mystiques qu'il a analysées, non leur foi !

Faut-il attribuer à ce même sens de la déformation chez $M$. Goulet ce long et fort inutile galimatias (cf. pp. 82 à 85 ) visant à 
nous montrer que la pensée sociale de Bergson s'exprime non seulement dans Les Deux Sources mais à travers toutes les grandes auvres de Bergson? Nous avons consacré trois chapitres de notre ouvrage à faire précisément cette preuve et à montrer que la philosophie sociale de Bergson est présente dans chacune de ses articulations fondamentales; qu'il s'agisse de la liberté, de l'intelligence, de l'intuition, du rire, du bon sens, de l'évolution biologique, du langage, etc. . ., partout se manifeste chez Bergson l'importance du social. Comme du reste pourrait-on prétendre à une compréhension de ces thèmes bergsoniens sans leur indispensable référence au social et comment a fortiori une interprétation de la philosophie sociale de Bergson pourrait-elle en toute naïveté " glisser prestement "sur des thèmes aussi fondamentaux ? Décidément, il y a lieu de s'interroger sur une façon aussi étrange de lire un texte et d'en tirer une conclusion si manifestement contraire à celle qui s'y trouve pourtant exprimée.

Que dire enfin de cette longue et incohérente digression sur Spencer (cf. pp. 88 à 91) ? Quelle en est la signification et la pertinence? Veut-on vraiment montrer en utilisant le cas Spencer "ce qu'il en coûte philosophiquement d'opter clairement pour la pure positivité " (p. 88)?

Bien mauvais exemple pour vouloir illustrer sans doute ce "terrible écueil" de la positivité que Bergson aurait su éviter, quand on considère que la critique de Bergson à l'endroit de Spencer vise justement à faire voir son manque de positivité qui l'a conduit au "fantôme " de la réalité ! Du reste, à quoi se réfère au juste, dans l'esprit de $M$. Goulet, ce spectre de la positivité qui suscite tant de phobies et qu'il utilise (c'est le moins qu'on puisse dire) dans un sens fort peu bergsonien?

Ou bien veut-on, en introduisant subrepticement l'exemple de Spencer, suggérer une lecture et une interprétation de la philosophie sociale de Bergson qui conserverait jusque dans ses ultimes développements la marque quasi indélébile d'une réaction antispencérienne de jeunesse, conçue comme unique et constant cadre de référence? C'est là prendre une voie facile puisque la référence à Spencer est tellement explicite chez Bergson ; c'est là, du même coup, s'engager dans une réduction simpliste qui mène forcément à la pensée résiduelle en même temps qu'elle discrédite l'intelligence du philosophe ; comme si Bergson, jusque dans les 
Remarques finales aux Deux Sources, en était resté à l'antispencérisme de ses vingt ans! Loin de nous l'intention qui voudrait nier ou minimiser l'importance du spencérisme pour comprendre Bergson; mais il y a bien davantage.

La référence aux idées sociologiques, anthropologiques et psychologiques les plus vives et les plus contemporaines de Bergson est autant sinon plus indispensable à une lecture sensée et honnête de la pensée sociale de Bergson.

On ne dénoncera avec jamais trop de vigueur les omissions en ce sens, car il en va de la compréhension fondamentale de la démarche bersonienne dans les questions sociales, comme il en va de la pertinence que Bergson a su donner à chacune de ses considérations touchant la société.

$\grave{A}$ qui veut feindre d'ignorer ce contexte ou se refuse d'en tenir compte sous le prétexte (difficilement conciliable du reste avec l'ouverture et la disponibilité de la recherche philosophique) de vouloir "protéger Bergson" de la positivité scientifique, il convient de rappeler la dernière proposition du Tractatus de Wittgenstein : "Ce dont on ne peut parler, il faut le taire."

Université d'Ottawa 